\title{
Toll-Interacting Protein, Tollip, Inhibits IL-13-Mediated Pulmonary Eosinophilic Inflammation in Mice
}

\author{
Yoko Ito $^{a}$ Niccolette Schaefer ${ }^{a}$ Amelia Sanchez $^{a}$ David Francisco ${ }^{b}$ \\ Rafeul Alam $^{a}$ Richard J. Martin ${ }^{a}$ Julie G. Ledford ${ }^{b}$ Connor Stevenson ${ }^{a}$ \\ Di Jiang ${ }^{\mathrm{a}}$ Liwu Lic Monica Kraft ${ }^{\mathrm{b}}$ Hong Wei Chu ${ }^{\mathrm{a}}$ \\ ${ }^{a}$ Department of Medicine, National Jewish Health, Denver, CO, ${ }^{b}$ Department of Medicine, University of Arizona \\ College of Medicine, Tucson, AZ, and ' Department of Biological Sciences, Virginia Polytechnic Institute and State \\ University, Blacksburg, VA, USA
}

\section{Keywords}

Tollip · Eosinophil · Eotaxin-2/CCL24 · Interleukin-13 · Interleukin-33 $\cdot$ ST2L

\begin{abstract}
Toll-interacting protein (Tollip) is a key negative regulator of innate immunity by preventing excessive proinflammatory responses. Tollip genetic variation has been associated with airflow limitation in asthma subjects and Tollip expression. Whether Tollip regulates lung inflammation in a type 2 cytokine milieu (e.g., IL-13) is unclear. Our goal was to determine the in vivo role of Tollip in IL-13-mediated lung eosinophilic inflammation and the underlying mechanisms. Tollip-knockout (KO) and wild-type (WT) mice were inoculated intranasally with recombinant mouse IL-13 protein to examine lung inflammation. To determine how Tollip regulates inflammation, alveolar macrophages and bone marrow-derived macrophages from Tollip KO and WT mice were cultured with or without IL-13 and/or IL-33. IL-13-treated Tollip KO mice significantly increased lung eosinophilic inflammation and eotaxin-2 (CCL24) levels compared with the WT mice. IL-13treated Tollip KO (vs. WT) macrophages, in the absence and
\end{abstract}

\section{KARGER}

() 2018 S. Karger AG, Basel

E-Mail karger@karger.com

www.karger.com/jin particularly in the presence of IL-33, increased expression of the IL-33 receptor ST2L and CCL24, which was in part dependent on enhanced activation of interleukin (IL)-1 receptorassociated kinase 1 (IRAK1) and signal transducer and activator of transcription 6 (STAT6). Our results suggest that Tollip downregulates IL-13-mediated pulmonary eosinophilia in part through inhibiting the activity of the ST2L/IL-33/IRAK1 axis and STAT6.

(c) 2018 S. Karger AG, Basel

\section{Introduction}

Asthma, a chronic inflammatory disease of the airways, has increased in prevalence and severity in industrialized nations over the past 50 years [1]. To date, 2 major endotypes of asthma have been described: type 2 (T2) high and T2 low. Patients with T2-high asthma have increased eosinophils in sputum and airway tissue [2]. Eosinophils exert toxic effects on host tissue and exaggerate inflammation through the release of a variety of inflammatory mediators [3]. Asthmatics with a higher number of eosinophils in blood and/or sputum seem to have

Prof. Hong Wei Chu

Department of Medicine, National Jewish Health

1400 Jackson Street, Room A639

Denver, CO 80206 (USA)

E-Mail chuhw@njhealth.org 
more severe disease and more frequent exacerbations than those with lower eosinophil counts $[4,5]$.

Major regulators of asthma, such as interleukin-33 (IL-33), stimulate the innate or adaptive immune system to secrete T2 cytokines, such as IL-13. Because of the extensive use of mouse IL-13 treatment models in asthma research [2, 6-8], IL-13 has been considered as one of the key regulators in eosinophil recruitment and survival, and other pathological features of asthma, such as airway fibrosis. One of the major mechanisms by which IL-13 induces airway eosinophilia is through the production of eotaxins. Although several types of lung cells produce eotaxins, alveolar macrophages (AMs), particularly in the presence of T2 cytokine stimulation, are critical in eotaxin production. AMs are involved in asthma pathogenesis in both humans and mice [9-11]. IL-13 as well as IL33 have been shown to increase the expression of eotaxin-2 (CCL24) and other chemokines, including CCL17, CCL18, and CCL22 [9]. However, the mechanisms regulating eotaxin-2 (CCL24) expression in macrophages exposed to $\mathrm{T} 2$ cytokines remain unclear.

Toll-interacting protein (Tollip) is a critical regulator of Toll-like receptor (TLR) and IL-1/IL-1 receptor (IL1R) signaling by interacting with IL-1R-associated kinase 1 (IRAK1) and subsequently suppressing IRAK1 activity [12]. Many types of cells, including macrophages and epithelial cells, express Tollip [13-15]. Although Tollip is classically considered as a negative regulator in bacterial infection to prevent excessive inflammation and tissue damage, its role in noninfectious processes has not been well understood. Several Tollip single nucleotide polymorphisms have been implicated in sepsis [16], tuberculosis [13], idiopathic pulmonary fibrosis [17], and atopic dermatitis [18]. Recently, we reported that asthma subjects carrying the Tollip rs5743899 AG/GG genotypes demonstrate greater airflow limitation as indicated by a lower $\mathrm{FEV}_{1} / \mathrm{FVC}$ ratio than those carrying the AA genotype [14]. Moreover, human airway epithelial cells with the AG/GG genotype express less Tollip protein, and produce more eotaxin-3 (CCL26) when stimulated with IL13 [14]. However, the in vivo role of Tollip in regulating IL-13-mediated lung inflammation, especially eosinophilic inflammation, is not clear.

In the present study, we hypothesized that Tollip deficiency in an in vivo T2 cytokine (e.g., IL-13) milieu enhances eotaxin production and excessive pulmonary eosinophilic inflammation. To test this hypothesis, we used a Tollip-deficient mouse model of IL-13 treatment to determine the role of Tollip in eotaxin production and pulmonary eosinophilia. Additionally, we examined the mechanisms by which Tollip downregulates eotaxin production by using cell culture models of AMs and bone marrow-derived macrophages (BMDMs) from Tollipdeficient mice.

\section{Methods}

Murine Model of IL-13-Induced Lung Inflammation

Tollip knockout (KO) and IRAK1 KO mice on the C57BL/6 background were obtained from Dr. Liwu Li at Virginia Polytechnic Institute and State University, and bred at National Jewish Health (NJH) Biological Research Center (BRC). ST2 KO mice on the BALB/c background were obtained from Dr. Rafeul Alam at NJH. Wild-type (WT) mice on the C57BL/6 and BALB/c background were purchased from the Jackson Laboratory and housed with the gene KO mice at NJH BRC under pathogen-free housing conditions. All of the experimental protocols were approved by the Institutional Animal Care and Use Committee at NJH. To establish an acute model of IL-13-induced airway disease, mice were intranasally instilled with 3 doses of IL-13 using modified methods as previously published $[19,20]$. Mice (13-17 weeks old, both females and males) were administered intranasally with recombinant mouse IL-13 (rmIL-13; R\&D Systems Inc., Minneapolis, MN, USA) at $500 \mathrm{ng} /$ mouse prepared in $0.1 \%$ BSA (bovine serum albu$\min$ ) or $0.1 \%$ BSA (control) once daily for 3 consecutive days. The mice were sacrificed 24 and $48 \mathrm{~h}$ after the last IL-13 treatment. Bronchoalveolar lavage (BAL) was performed with $1 \mathrm{~mL}$ of saline. BAL cells were used for the leukocyte count, and BAL fluid (BALF) was saved for ELISA.

\section{Mouse Lung Histopathologic Analysis}

The apical lobe of the mouse right lung was fixed in $10 \%$ formalin, embedded in paraffin, and cut at 5- $\mu \mathrm{m}$ thickness for histopathologic analysis. Lung sections were stained with hematoxylin and eosin (HE), and evaluated in a blinded fashion under a light microscope using a histopathologic inflammatory scoring system as we described previously [21]. A final score per mouse on a scale of 0-26 (least to most severe) was obtained based on an assessment of the quantity and quality of peribronchiolar and peribronchial inflammatory infiltrates, luminal exudates, perivascular infiltrates, and parenchymal pneumonia.

\section{Murine Macrophage Culture}

Murine primary AMs were obtained by lavaging the lungs. AMs were isolated by adherence and cultured in DMEM (Dulbecco's Modified Eagle's Medium) supplemented with 10\% FBS (fetal bovine serum) and penicillin/streptomycin. At 6-8 h after adherence in 96-well culture plates, AMs were stimulated with rmIL-13 (10 ng/mL) \pm rmIL-33 (10 ng/mL; Pepro Tech, Rocky Hill, NJ, USA). Cell culture supernatants were collected for CCL24 protein measurement. Cells were saved in RLT lysis buffer (Qiagen Inc., Germantown, MD, USA) at $-80^{\circ} \mathrm{C}$ for subsequent total RNA extraction and RT-PCR.

Due to the limited number of AMs available for the mechanistic studies, BMDMs were used to study how Tollip regulates CCL24 production in the presence or absence of IL-13 and/or IL33. Bone marrow cells from Tollip KO (C57BL/6 background), ST2 KO (BALB/c background), IRAK1 KO (C57BL/6 back- 
ground), and WT (C57BL/6 and BALB/c) mice were cultured in DMEM supplemented with $10 \%$ FBS, penicillin/streptomycin and recombinant mouse macrophage colony-stimulating factor 25 $\mathrm{ng} / \mathrm{mL}$; R\&D Systems Inc.) for 7 days to differentiate them into BMDMs. On day 7, BMDMs were harvested by trypsinization and then seeded at $2 \times 10^{5}$ cells/well onto 24 -well cell culture plates. On day 8 , cells were stimulated by rmIL-13 \pm rmIL-33, and in some conditions cells were preincubated with an anti-mouse ST2L/IL$33 \mathrm{R}$ antibody (R\&D Systems Inc.) or the rat IgG2B isotype control (R\&D Systems Inc.) for $2 \mathrm{~h}$. At the indicated time points, culture medium and cells were collected for protein and mRNA determination.

\section{ELISA}

A murine CCL24 ELISA kit (R\&D Systems Inc.) was used to quantify CCL24 in murine BALF and macrophage culture medium. Murine IL-33 ELISA (R\&D Systems Inc.) was used to measure IL-33 in BALF. The detection range for CCL24 and IL-33 was from 15.6 to $1,000 \mathrm{pg} / \mathrm{mL}$.

\section{Quantitative Real-Time PCR}

Quantitative real-time PCR was conducted using Taqman Gene Expression Assay (Applied Biosystems, Foster City, CA) to determine relative mRNA levels of ST2L and IL-33. Target gene expression was normalized by the housekeeping gene $18 \mathrm{~S}$ rRNA. The comparative threshold cycle method $\left(\Delta \Delta \mathrm{C}_{t}\right)$ was applied to determine the relative levels of target genes.

\section{Western Blot Analysis}

Equal amounts of protein from samples with different treatments were separated on 10 or $15 \%$ SDS-PAGE, transferred onto polyvinylidene difluoride membranes, and probed with a goat anti-Tollip antibody (Santa Cruz Biotechnology Inc., Dallas, TX, USA), a rabbit anti-IRAK1 antibody (Cell Signaling Technology Inc., Danvers, MA, USA), a rabbit anti-pIRAK1 antibody (Phospho-Thr209; Aviva Systems Biology, San Diego, CA, USA), a goat anti-ST2L antibody (R\&D Systems Inc.), a rabbit anti-STAT6 or anti-pSTAT6 (Phospho-Tyr641, Cell Signaling Technology Inc.) antibody, or a mouse anti-GAPDH antibody (Santa Cruz Biotechnology Inc.).

Blots were then incubated with appropriate horseradish peroxidase-linked secondary antibodies and developed with ECL Western blotting substrate. Densitometry was performed using the NIH Image-J software. Activation of IRAK1 or STAT6 was measured by analyzing their phosphorylation levels as indicated by the ratio of phosphorylated versus nonphosphorylated IRAK1 or STAT6.

\section{Statistical Analysis}

If the data were normally distributed, 1-way analysis of variance (ANOVA) followed by a Tukey post hoc test was used for multiple comparisons. The Student $t$ test was used when 2 groups were compared. For nonparametric data, the KruskalWallis test for multiple comparisons or the Mann-Whitney test for 2 group comparisons were applied. Pearson correlation analysis was performed to determine the relationship of lung eosinophils and CCL24 levels. A $p$ value $<0.05$ was considered significant.

\section{Results}

Tollip Inhibits Eosinophil Recruitment into the Lung of IL-13-Treated Mice

After $24 \mathrm{~h}$ of the last IL-13 treatment, the total leukocyte counts in BALF were increased in both WT and Tollip KO mice, and were higher in Tollip KO mice than WT mice, although a significant difference was not achieved $(p=0.06$; Fig. 1a). Importantly, following IL-13 treatment, the percentage and number of eosinophils in BALF were significantly higher in Tollip KO mice than WT mice (Fig. 1b, c). Additionally, lymphocytes were also increased in Tollip KO mice as compared to the WT mice (Fig. 1d). IL-13 increased the number of neutrophils in both WT and Tollip KO mice, but there was no significant difference between the 2 groups (Fig. 1e). The number of macrophages in BALF did not change after IL-13 exposure (Fig. 1f). Examination of lung histopathology of IL13-treated Tollip KO mice revealed more severe infiltrate of inflammatory cells including eosinophils around large airways and in the alveolar space (Fig. 2a-f). The histopathology score, an indication of overall inflammation levels, was significantly higher in IL-13-treated Tollip KO mice than the WT mice (Fig. 2g).

We also collected BALF at $48 \mathrm{~h}$ after the last IL-13 treatment. Tollip KO mice showed higher eosinophil and lymphocyte counts in BALF than WT mice, but the differences did not reach the statistically significant level (eosinophils - WT: 40,311 \pm 9,905 cells/mL, KO: 110,574 \pm 26,881 cells/mL, $p=0.07$; lymphocytes - WT: 4,215 \pm 1,119 cells/mL, KO: $16,712 \pm 5,172$ cells $/ \mathrm{mL}, p=0.13$ ).

\section{Tollip Reduces Lung Secretion of CCL24 into BALF}

As the focus of our study was to investigate how Tollip regulates eosinophilic inflammation, our mechanistic studies were centered on the pathways related to eotaxin expression, particularly at the earlier time point (e.g., $24 \mathrm{~h}$ ) to mimic acute inflammation related to asthma exacerbations. In line with the BAL eosinophil data, IL13-treated Tollip KO mice showed higher levels of CCL24 protein in BALF than the WT mice at $24 \mathrm{~h}$ (Fig. 3a) and at $48 \mathrm{~h}$ (WT: $638 \pm 256 \mathrm{pg} / \mathrm{mL}, \mathrm{KO}: 1,654 \pm 397 \mathrm{pg} / \mathrm{mL}$, $p=0.06)$. Notably, CCL24 protein levels were positively correlated with BAL eosinophil counts in both Tollip KO and WT mice (Fig. 3b).

\section{Tollip Suppresses IL-13-Induced CCL24 in Cultured Murine Macrophages}

As CCL24 produced by macrophages is responsible for IL-13-mediated airway eosinophilia in a murine model 


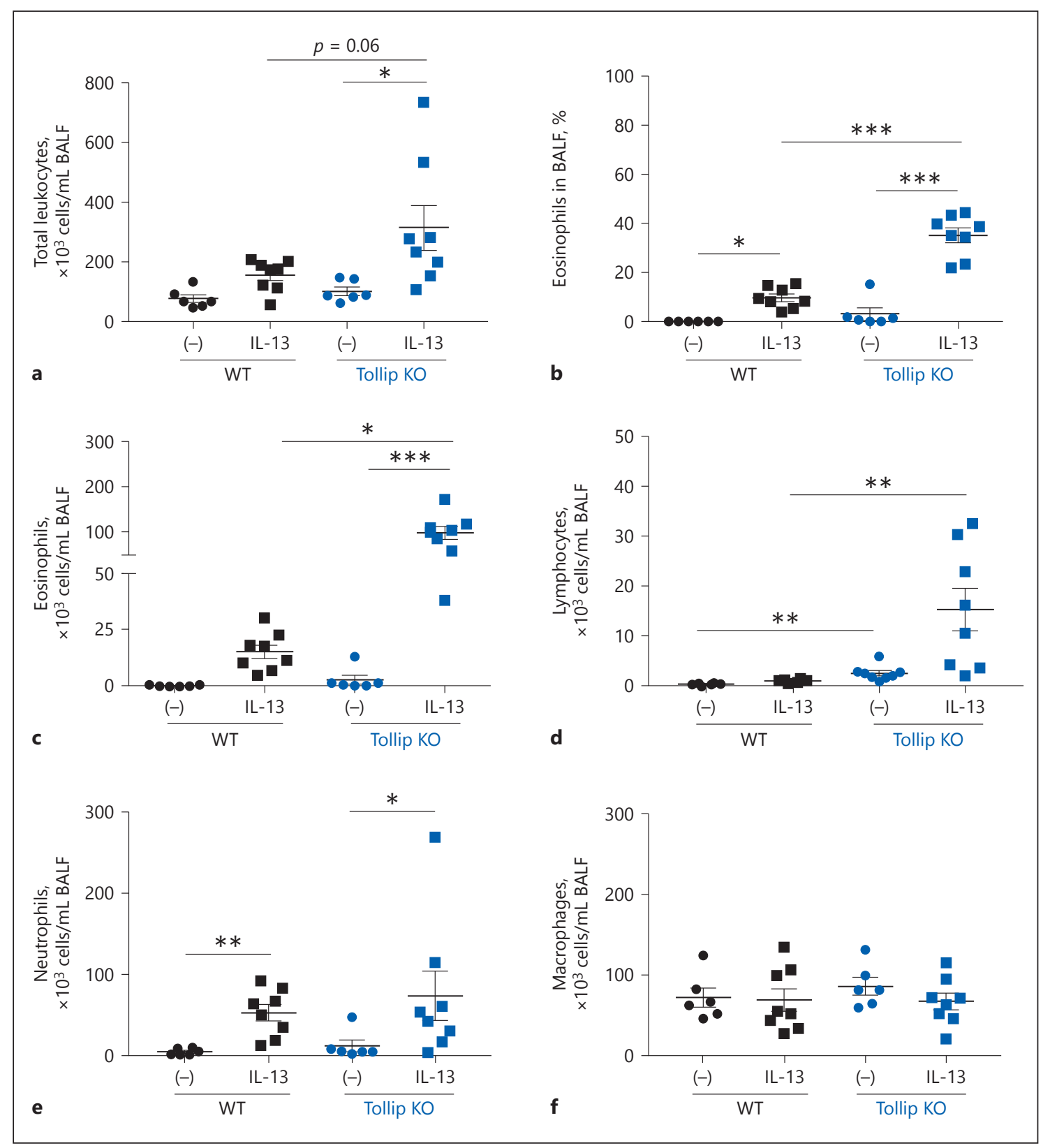

Fig. 1. Tollip KO mice treated with IL-13 show exaggerated eosinophil recruitment into the airways. Tollip KO and WT mice were treated with recombinant mouse IL-13 and sacrificed $24 \mathrm{~h}$ after the last IL-13 treatment. BALF was collected for counting the total numbers of leukocytes (a), eosinophils (b, c), lymphocytes (d), neutrophils (e), and macrophages (f). $n=6-8$ mice/group. Data are expressed as means \pm SEM. ${ }^{*} p<0.05,{ }^{* *} p<0.01$, and $* * * p<0.001$.

[7], we determined the impact of Tollip expression on CCL24 secretion by macrophages. To test if IL-13-exposed AMs from Tollip KO mice produce more CCL24 than the WT cells, AMs were treated with rmIL-13. After IL-13 treatment, AMs from Tollip KO mice showed sig- nificantly higher levels of CCL24 secretion than the WT mice (Fig. 3c).

As further mechanistic studies in murine AMs were difficult to pursue due to the limited number of cells, we utilized BMDMs from Tollip KO and WT mice. We con- 


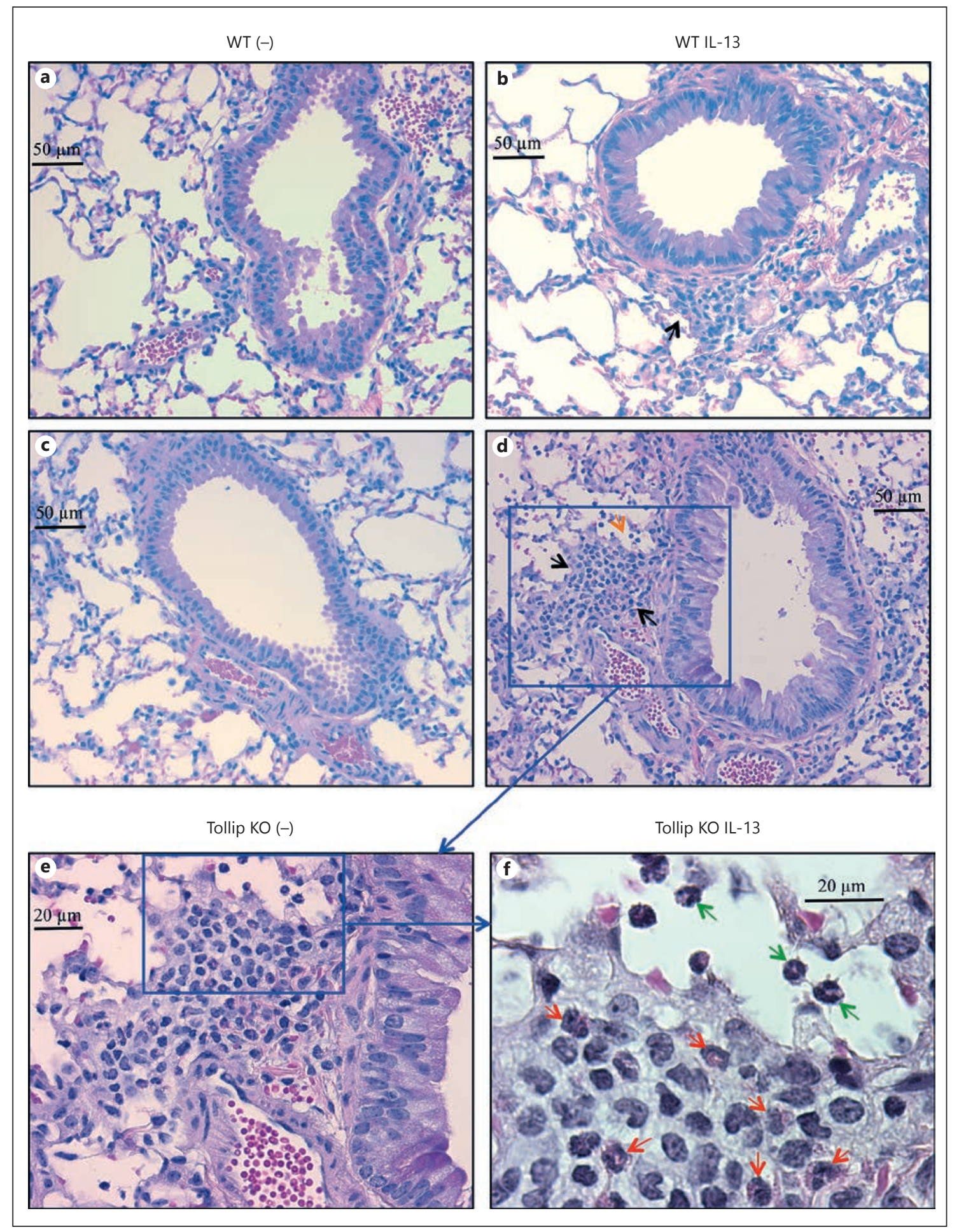

Fig. 2. Tollip KO mice treated with IL-13 show increased lung tissue inflammation. Tollip KO and WT mice were treated with recombinant mouse IL-13 and sacrificed $24 \mathrm{~h}$ after the last IL-13 treatment. Histopathologic images of HE-stained lung tissues are from WT $(\mathbf{a}, \mathbf{b})$ and Tollip $\mathrm{KO}(\mathbf{c}, \mathbf{d})$ mice. Black and orange arrows indicate leukocyte infiltrate around an airway and in the alveolar space, respectively. To further identify inflammatory cell types in panel d (original magnification, $\times 200$ ), higher magnification images of the framed inflammation area are shown in panel $\mathbf{e}(\times 400)$ and panel $\mathbf{f}(\times 1,000)$. The red arrows indicate eosinophils around the airway, while the green arrows indicate eosinophils in the alveolar space (f). $g$ Histopathology score. $n=6-8$ mice/group. Data are expressed as means \pm SEM. ${ }^{* *} p<0.01$ and ${ }^{* * *} p<0.001$.

(Figure continued on next page.) 


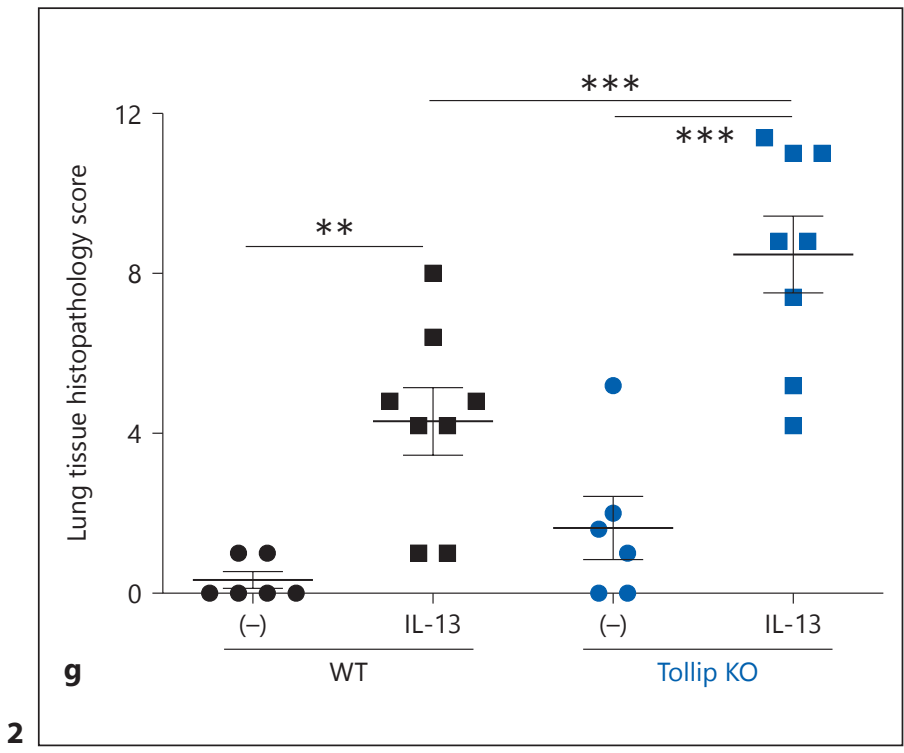

firmed that IL-13-treated BMDMs from Tollip KO mice produced higher levels of CCL24 protein than those from the WT mice (Fig. 3d), which was consistent with the data in cultured AMs and BALF in vivo.

Tollip Decreases IL-13-Induced ST2L Expression and IL-13/IL-33-Mediated CCL24 Production in Murine BMDMs

How Tollip regulates CCL24 expression under IL-13 stimulation remains unclear. A previous study using the BMDM culture system demonstrated the synergic effects of IL-13 in combination with IL-33, another key cytokine in the T2 inflammation, on eotaxin expression, which is largely dependent on the induction of the IL-33 receptor ST2L by IL-13 [22]. Importantly, the ST2L protein level was significantly higher in IL-13-treated BMDMs from the Tollip KO than the WT mice (Fig. 4a). As various cell types in the lung constitutively express IL-33, our data suggest that ST2L upregulation associated with Tollip deficiency may enhance the crosstalk between IL-13 and IL33 pathways, and further increase eotaxin expression and eosinophilic inflammation. Therefore, we stimulated BMDMs with IL-13 and IL-33 simultaneously and measured CCL24 secretion. IL-13 and IL-33 stimulation in Tollip KO mouse BMDMs synergistically increased CCL24 secretion, which was markedly (about 6 -fold) inhibited by a ST2L-neutralizing antibody (Fig. $4 \mathrm{~b}$ ). The contribution of ST2L to IL-13- and IL-33-mediated CCL24 expression was further revealed by the finding that ST2L KO BMDMs produced significantly less CCL24

Tollip Inhibits Lung Eosinophilic Inflammation than the WT cells (Fig. 4c). Moreover, as previous reports demonstrated that IL-33 amplifies IL-13-induced ST2L expression [22], we examined whether Tollip deficiency enhances ST2L induction by IL-13 and IL-33. As shown in Figure 4d, ST2L mRNA and protein were markedly induced by IL-13 and IL-33 in BMDMs from the Tollip KO mice.

\section{Tollip Downregulates IL-13/IL-33-Induced CCL24 in}

Part by Inhibiting IRAK1 Activation

IL-33 is a member of the IL-1 family. Upon IL-33 stimulation, MyD88 (myeloid differentiation primary response protein 88), IRAK1, IRAK4, and TRAF6 (tumor necrosis factor receptor-associated factor 6) are all recruited to ST2L, which activates MAP kinase and nuclear factor (NF) $-\kappa B$ [23]. Tollip may negatively regulate the IL-33 signaling pathway by inhibiting the activation of IRAK1 [24]. To determine if CCL24 induction by IL-13 and IL-33 is regulated through IRAK1 activation, we measured CCL24 production in IL-13- and IL-33-stimulated BMDMs of IRAK1 KO and WT mice. The dependence of CCL24 expression on IRAK1 was supported by the lack of CCL24 production in IL-13- and IL-33-stimulated BMDMs from IRAK1 KO mice as compared to the WT mice (Fig. 5a). Notably, Tollip KO BMDMs with IL13 and IL-33 stimulation showed greater IRAK1 activation than WT cells (Fig. 5b), supporting Tollip's inhibitory effect on IRAK1 activation and subsequent CCL24 production. Having shown the inhibitory role of Tollip in IRAK1 activation, we determined if Tollip inhibited STAT6 activation, a pathway involved in IL-13-induced ST2L expression [25]. STAT6 activation was compared in BMDMs of WT and Tollip KO mice at 60 min after IL-13 and IL-33 costimulation to mimic the coexistence of these 2 cytokines in our IL-13-treatment mouse model. We found that STAT6 activation was moderately higher in BMDMs of Tollip KO mice than WT mice (Fig. 5c), suggesting an inhibitory role of Tollip in the early IL-13 signaling event.

\section{IL-33 Protein Is Constitutively Secreted into the}

Mouse Airway, and Tollip Inhibits IL-33 mRNA

Induction by IL-13 in AMs

As BMDMs from the Tollip KO mice increased CCL24 secretion after IL-13 and IL-33 costimulation, we sought to determine if IL-33 protein levels in BALF were different between WT and Tollip KO mice. Although IL-33 protein was detectable in the BALF of control mice, it was not further induced by IL-13 treatment (Fig. 6a) in WT or Tollip KO mice. As the murine macrophage is 
one of the major IL-33 sources [26-30], we examined whether IL-13 alone induced IL-33 expression in AMs. IL-33 mRNA was significantly induced by IL-13 in Tollip KO AMs, but not in WT AMs. The induction of IL-33
mRNA by IL-13 tended to be greater in AMs from the Tollip KO mice than the WT mice (Fig. 6b). However, IL33 protein in culture medium from IL-13-treated AMs was undetectable by ELISA. AMs were stimulated with

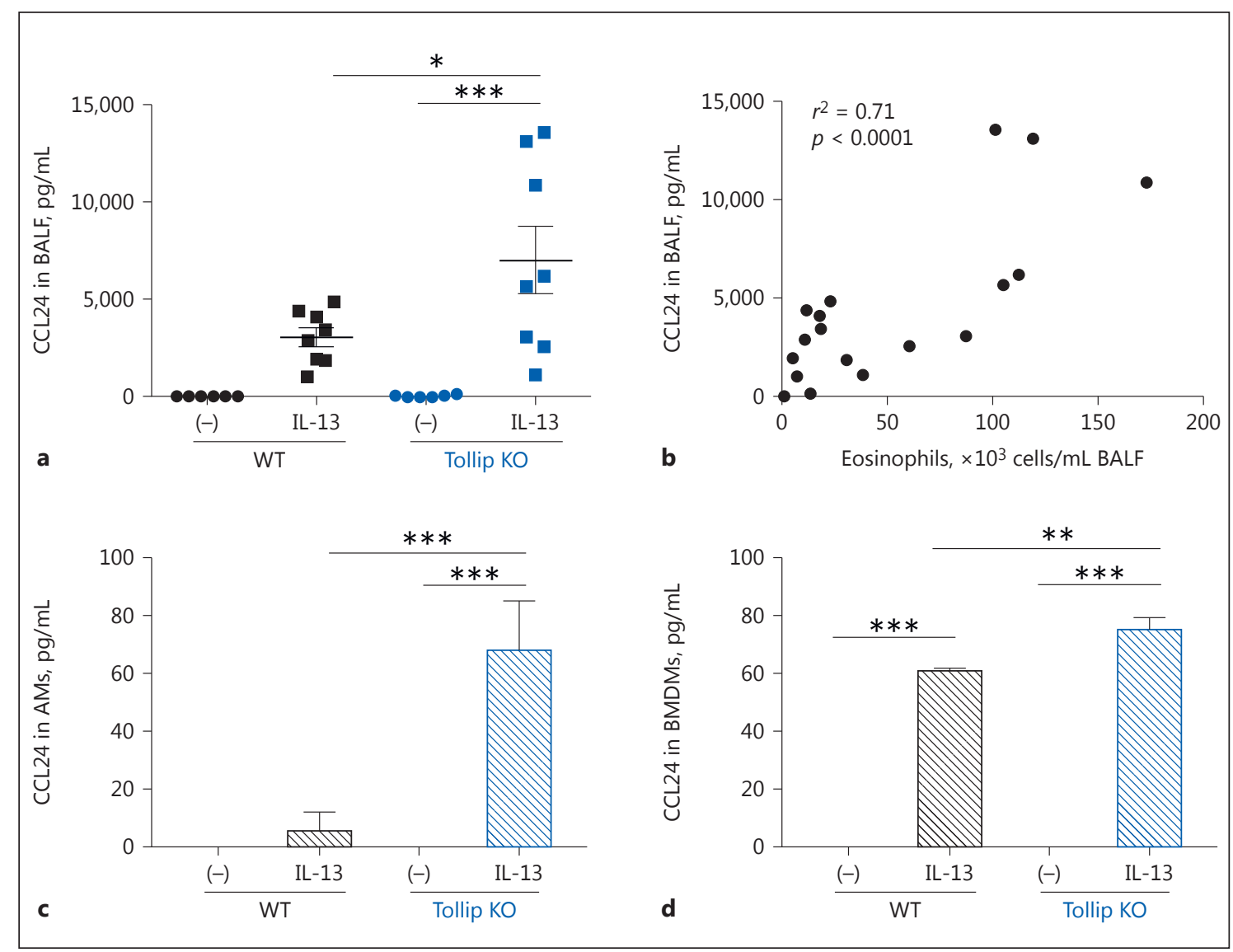

Fig. 3. Tollip deficiency enhances IL-13-induced CCL24 production in mouse BALF and macrophages. Tollip KO and WT mice were treated with IL-13 and sacrificed $24 \mathrm{~h}$ after the last IL- 13 treatment. a CCL24 protein levels in BALF. $n=6-8$ mice/group. $\mathbf{b}$ Scatterplot showing a positive correlation between the number of eosinophils and the level of CCL24 protein in BALF. C CCL24 protein levels in culture medium of Tollip KO and WT mouse AMs stimulated with IL-13 for 48 h. $n=6-8$ replicates from 2 independent experiments. d CCL24 protein measured in culture medium $( \pm$ IL-13 for $24 \mathrm{~h}$ ) of BMDMs from Tollip KO and WT mice. $n=9$ replicates from 3 independent experiments. Data are expressed as means \pm SEM. ${ }^{*} p<0.05,{ }^{* *} p<0.01$, and ${ }^{* * *} p<0.001$.
Fig. 4. Tollip deficiency increases IL-13-induced ST2L expression, and CCL24 production by IL-13 and IL-33 in murine BMDMs. a BMDMs from Tollip $\mathrm{KO}$ and WT mice were incubated with IL-13 for 24 h: a representative Western blot of IL-13-induced ST2L protein expression (left); densitometric analysis of ST2L protein (right). b CCL24 protein levels in culture medium of Tollip $\mathrm{KO}$ and WT mouse BMDMs stimulated with IL-13, IL-33, and a combination of IL-13 and IL-33 in the presence or absence of a ST2L neutralizing antibody (ST2L ab) or the IgG2B isotype control
(IgG) for 24 h. c CCL24 protein levels in culture medium of ST2 KO and WT mouse BMDMs stimulated with IL-13 and IL-33 for 48 h. d BMDMs from Tollip KO and WT mice were cultured with IL-13 and/or IL-33 for 24 h: ST2L mRNA relative levels (left); a representative Western blot of ST2L protein and densitometric analysis (right). Data are expressed as means \pm SEM. $n=9$ replicates from 3 independent experiments. ${ }^{*} p<0.05$, ${ }^{* *} p<0.01$, and $* * * p<0.001$.

(For figure see next page.) 


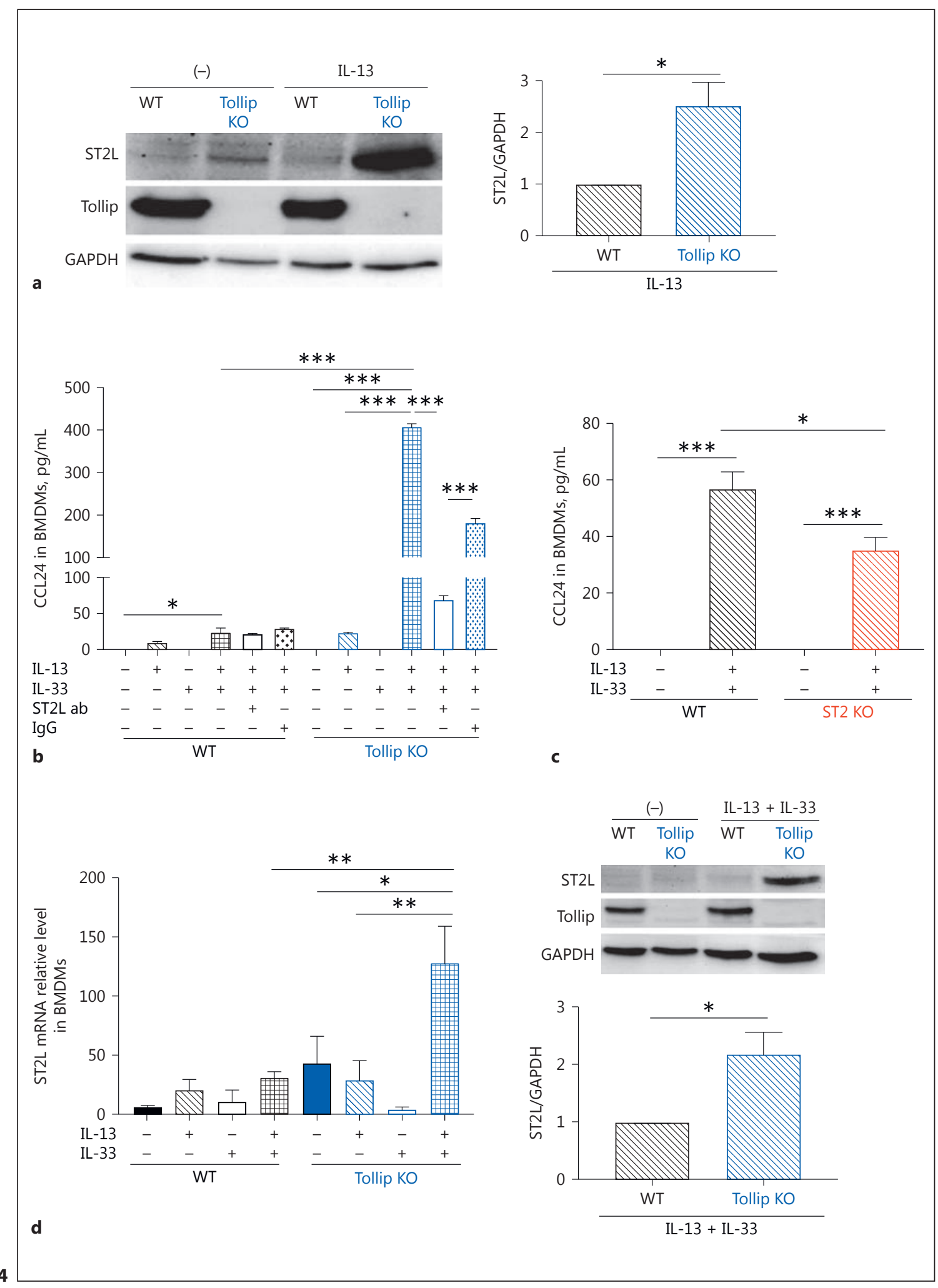




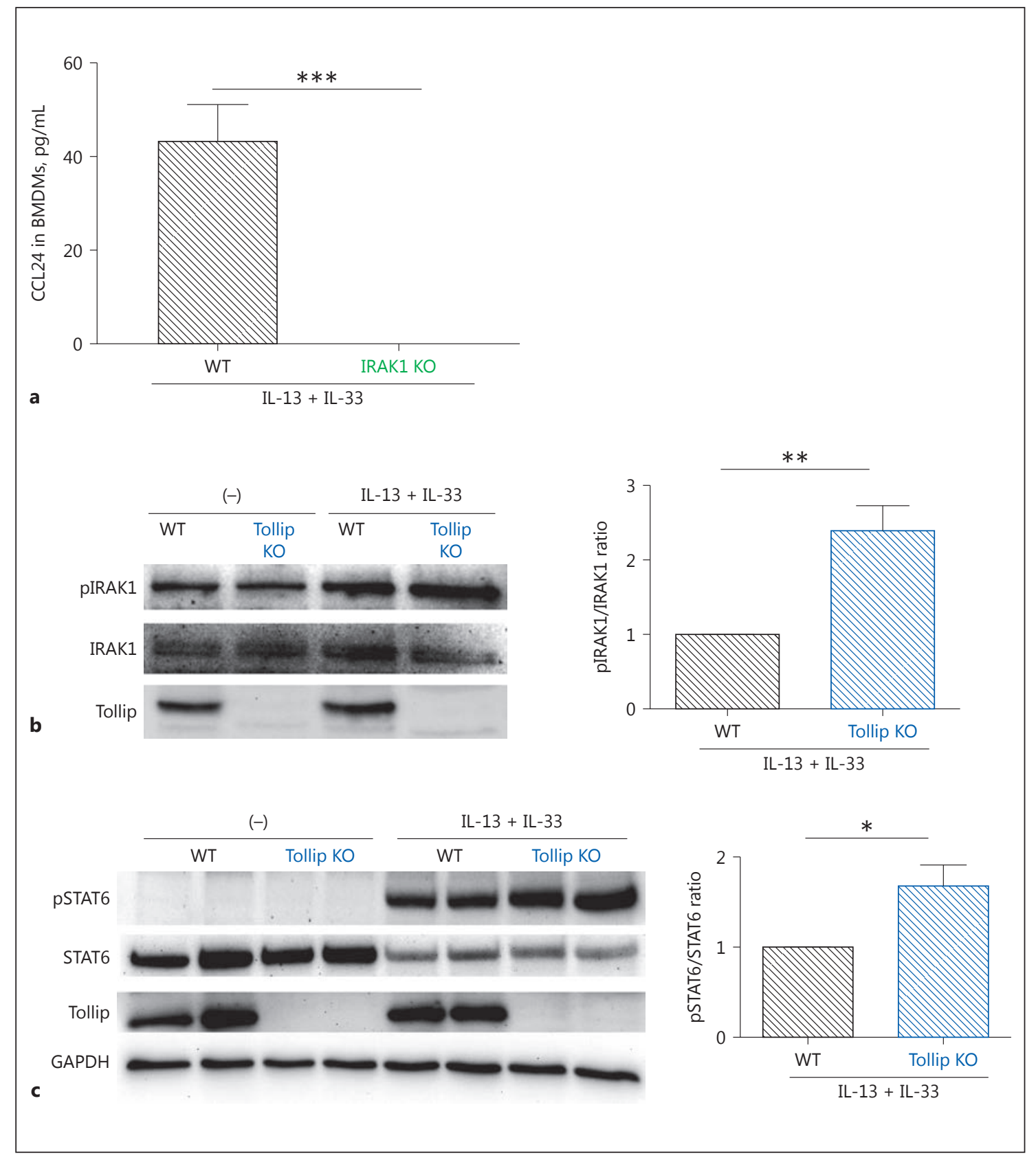

Fig. 5. Role of IRAK1 and STAT6 in CCL24 induction by IL-13 and IL-33 in murine BMDMs. a CCL24 protein levels in culture medium of IRAK1 KO and WT mouse BMDMs stimulated with IL-13 and IL-33 for 48 h. $n=9$ replicates from 3 independent experiments. b BMDMs from Tollip KO and WT mice were incubated with IL-13 and IL-33 for 24 h: a representative Western blot of phosphorylated IRAK1 (pIRAK1) and total IRAK1 proteins (left); densitometric analysis of IRAK1 activation, as determined by the ratio of pIRAK1 to total IRAK1 (right). $n=9$ replicates from 3 independent experiments. c Representative Western blot of phosphorylated STAT6 (pSTAT6) and STAT6 in Tollip KO and WT mouse BMDMs stimulated with IL-13 and IL-33 for 60 min (left); densitometric analysis of STAT6 activation, as determined by the ratio of pSTAT6 to total STAT6 (right). $n=3$ replicates from 2 independent experiments. Data are expressed as means \pm SEM. ${ }^{*} p<0.05,{ }^{* *} p<0.01$, and ${ }^{* * *} p<0.001$. 


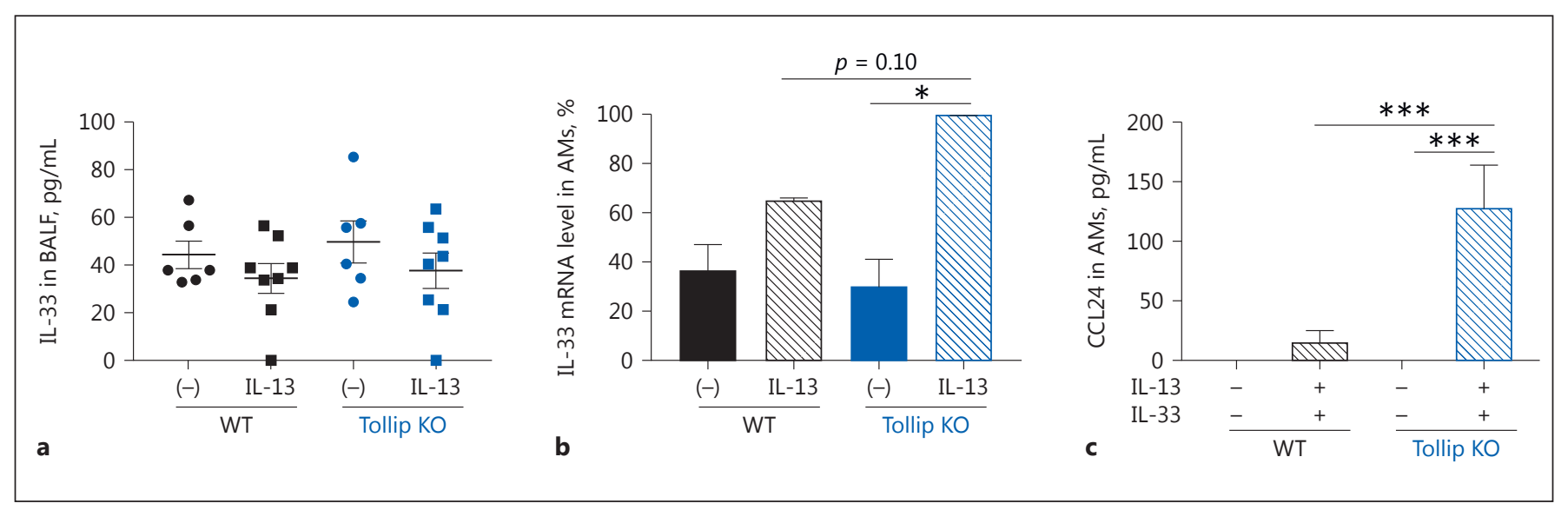

Fig. 6. IL-33 expression in mouse BALF and AMs. a Tollip KO and WT mice were treated with IL-13 and sacrificed $24 \mathrm{~h}$ after the last IL-13 treatment. IL-33 protein in BALF was measured by ELISA. $n=6-8$ mice/group. b AMs from Tollip KO and WT mice were incubated with IL-13 for $48 \mathrm{~h}$. IL-33 mRNA levels were expressed as the percentage of IL-13-treated AMs from the Tollip KO mice. c CCL24 protein levels in culture medium of Tollip KO and WT mouse AMs with or without stimulation of IL-13 and IL-33 for 48 h. Data are expressed as means \pm SEM. $n=6-9$ replicates from 2 or 3 independent experiments for culture of AMs. ${ }^{*} p<0.05$ and *** $p<0.001$.
Fig. 7. Proposed mechanisms whereby Tollip attenuates CCL24 production and pulmonary eosinophilic inflammation. In lung cells such as macrophages, IL-13 induces CCL24 expression in part through activating STAT6. Importantly, STAT6 activation will subsequently increase the expression of IL-33 receptor ST2L, which binds the preexisting (constitutive) IL-33 in the lung to further induce CCL24 expression and eosinophilic inflammation in part through activating IRAK1. Tollip inhibits CCL24 and eosinophilic inflammation by suppressing the activity of IRAK1 as well as STAT6.

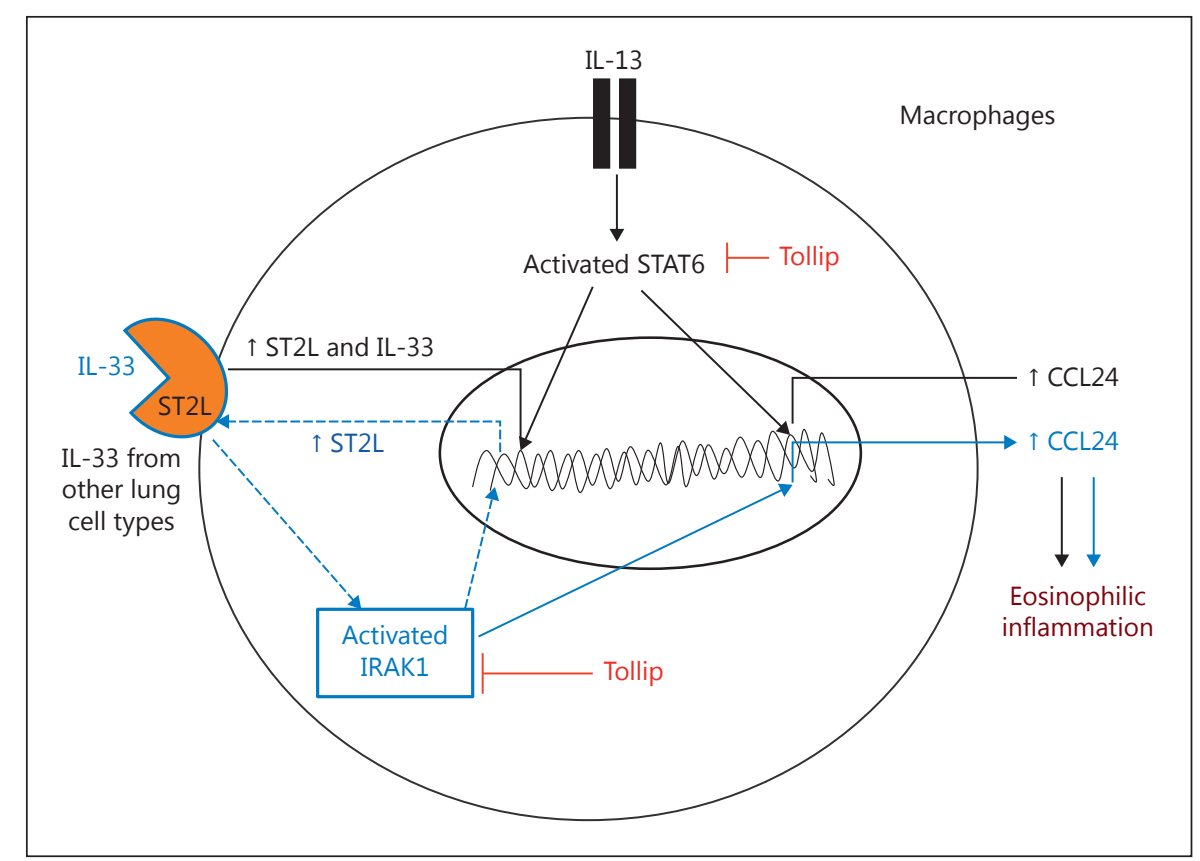

both IL-13 and IL-33 to mimic the coexistence of these 2 cytokines in our IL-13-treatment mouse model. Indeed, AMs from the Tollip KO mice, as compared to the WT mice, increased the production of CCL24 (Fig. 6c), which was consistent with our data obtained from BMDMs (Fig. 4b).

Tollip Inhibits Lung Eosinophilic Inflammation

\section{Discussion}

This is the first report demonstrating the regulation of in vivo lung eosinophilic inflammation by Tollip in the context of T2 cytokine treatment. Our results suggest that Tollip deficiency promotes pulmonary eosinophilia by enhancing CCL24 production. Major findings in this 
study include: (1) IL-13-treated Tollip KO mice show exaggerated pulmonary eosinophilia and CCL24 production, and (2) Tollip KO murine macrophages stimulated with IL-13 alone or in combination with IL-33 are characterized by enhanced ST2L expression, IRAK1 activation, and CCL24 production.

In this report, we clearly demonstrated that Tollip deficiency enhances IL-13-induced pulmonary eosinophilia along with CCL24 secretion into the airway lumen. Although various mediators such as eotaxins and IL-5 are known to promote murine lung eosinophilic inflammation, how these mediators are regulated under a T2 cytokine milieu remains poorly understood. In mouse lungs, AMs, in response to IL-13, serve as a major source of CCL24 that contributes to sustained recruitment of eosinophils into the airway lumen [7]. Indeed, we found that CCL24 production is higher in murine macrophages (AMs and BMDMs) from the Tollip KO mice than the WT mice. Once IL-13 binds to IL-13Ra1, STAT6 is activated and translocated to the nucleus where CCL24 transcription is upregulated [6]. We determined whether Tollip modifies the activation of STAT6, and observed marginal enhancement of STAT6 activation by IL-13 in BMDMs from the Tollip KO mice, suggesting that Tollip may downregulate CCL24 in part through reducing STAT6 activation. While we do not know how Tollip exactly affects STAT6 activation, Tollip is known to modify several signaling pathways (e.g., IL-1 $\beta$ R and TGF- $\beta$ R) by promoting degradation of ubiquitin-conjugated proteins [31-33]. Therefore, we propose that Tollip regulates the IL-13-STAT6 signaling pathway possibly by interacting with STAT6, but this awaits further confirmation.

One innovative aspect of our current study is to unravel the role of Tollip in inhibiting IL-13-mediated upregulation of the IL-33 receptor ST2L. IL-33, a member of the IL-1 cytokine family, is involved in asthma airway inflammation [34]. The binding of IL-33 to ST2L leads to IL-1R accessory protein (IL-1Racp) recruitment and the formation of heterodimeric signaling complex that involves MyD88, IRAK1, IRAK4, and TRAF6, and the activation of mitogen-activated protein kinase and NF- $\mathrm{KB}$ [35]. ST2L expression is higher in endobronchial biopsies of severe asthmatics than healthy controls and mild/moderate asthmatics, and associated with multiple T2-high asthma biomarkers, including blood eosinophils and eotaxin mRNA expression [36]. Notably, ST2L is induced by IL-13 in part through the STAT6 pathway $[22,25]$. Therefore, IL-33 has been shown to amplify CCL24 production in IL-13-pretreated macrophages [22]. As Tollip is a negative regulator for TLR and IL-1R pathways by inhibiting IRAK1 activation [24], we tested whether Tollip regulates the IL-13/IL-33/ST2L pathway, which was never evaluated before. Our data have extended previous reports [22] in that IL-13, particularly in the presence of IL-33, significantly increases ST2L expression and CCL24 production in murine macrophages. Our study demonstrates for the first time that Tollip deficiency leads to a greater induction of ST2L expression by IL-13 and/or IL33 in macrophages. Moreover, we have found that Tollip deficiency results in greater induction of CCL24 that is dependent on ST2L as Tollip's inhibitory effect on CCL24 is partially abrogated by an ST2L-neutralizing antibody. Thus, greater ST2L expression on macrophages from Tollip KO mice renders the cells more sensitive to the stimulation of IL-33 that is constitutively expressed in the lung and can be further upregulated by IL-13 treatment $[26,37]$. We are aware that, unlike the previous study [22], we did not observe a synergistic effect of IL-13 and IL-33 stimulation on ST2L expression in BMDMs from the WT mice. This may be explained by the use of a higher dose of IL-33 $(20 \mathrm{ng} / \mathrm{mL})$ in the previous study, and a lower dose of IL-33 (10 ng/mL) in our current study.

Having shown that IL-33/ST2L signaling is involved in CCL24 production, we further evaluated the role of the downstream signaling event of this pathway in eotaxin expression in the context of Tollip. IRAK1 is an integral component of the IL-33/ST2L signaling, but its contribution to IL-13/IL-33-mediated eotaxin production is unknown. Our data suggest that Tollip deficiency allows greater IRAK1 activation, and that IRAK1 deficiency significantly diminishes IL-13/IL-33-induced CCL24 expression. Together, our data indicate that Tollip acts on several checking points to downregulate CCL24 expression in the IL-13/IL-33 setting. The relative contribution of these checking points to Tollip's effects on eotaxin expression deserves further investigation.

Lung macrophages and epithelial cells produce IL-33 as an alarmin [26]. IL-33 is induced in AMs of ovalbumin-challenged mice $[28,38]$. Intriguingly, we did not see increased IL-33 in BALF of IL-13-treated Tollip KO mice as compared to the WT mice. Notably, in cultured AMs from the Tollip KO mice but not the WT cells, there was significant induction of IL-33 mRNA by IL-13. However, IL-33 protein was not detectable in AM supernatants. Together, our findings suggest that Tollip has a minimal impact on IL-33 protein release during its regulation of lung eosinophilic inflammation. Instead, as described in Results, IL-33 is constitutively released into the lung (e.g., airway lumen). We propose that greater induction of ST2L expression in lung cells (e.g., macrophages) of 
Tollip KO mice by IL-13 would increase the sensitivity of cells responding to endogenous/local IL-33, thereby promoting eosinophilic inflammation.

There are several limitations to the current study. First, because gene knockdown in human primary macrophages is very challenging, we were not able to knockdown Tollip in human primary AMs in order to translate our findings from mice to humans. Second, although lymphocyte recruitment is increased in Tollip KO versus WT mice, we have not assessed its contribution to eosinophilic inflammation. Recent studies have indicated that T2 innate lymphoid cells (ILC2s) contribute to the persistence of airway eosinophilia in patients with severe asthma through localized production of IL-13 [39]. Therefore, dissecting the role of Tollip in regulating ILC2s would further reveal how Tollip affects lung eosinophilic inflammation. Finally, our mechanistic study was focused on macrophages. In our pilot study, we examined if airway epithelial cells are involved in Tollip's function related to eosinophilic inflammation. As CCL24 levels in cultured mouse tracheal epithelial cells were not detectable, we did not pursue the epithelial model for the mechanistic studies. However, future studies are warranted to determine the role of Tollip in regulating eotaxin expression by primary human airway epithelial cells.

In Figure 7, we propose the novel mechanisms by which Tollip inhibits pulmonary eosinophilic inflam- mation. Briefly, IL-13-stimulated macrophages increase ST2L in part through STAT6 activation, and then CCL24 expression in part through IRAK1 activation in the context of lung constitutive and perhaps inducible IL-33 secretion. Tollip attenuates the activity of the IL-13/STAT6/ ST2L/IL-33/IRAK1/eotaxin axis in part by inhibiting STAT6 as well as IRAK1 activation. As our previous study demonstrated that asthmatics carrying the Tollip rs5743899 AG/GG genotype (vs. the AA genotype) express less Tollip protein and greater airflow limitation (less $\mathrm{FEV}_{1} / \mathrm{FVC}$ ), our current study likely offers an innovative and more precise approach to reducing airway inflammation in asthma patients. Enhancing Tollip expression or suppressing IRAK1 activation in asthmatics carrying the rs5743899 AG or GG genotype may effectively reduce airway inflammation and improve pulmonary function.

\section{Acknowledgements}

This work was supported by grants from the National Institutes of Health, R01 HL122321, R01 AI106287, R01 HL125128, and U19AI125357.

\section{Disclosure Statement}

The authors declare no competing financial interests.

\section{References}

1 Eder W, Ege MJ, von Mutius E: The asthma epidemic. N Engl J Med 2006;355:2226-2235.

2 Tabatabaian F, Ledford DK, Casale TB: Biologic and new therapies in asthma. Immunol Allergy Clin North Am 2017;37:329-343.

3 Carr TF, Berdnikovs S, Simon HU, Bochner BS, Rosenwasser LJ: Eosinophilic bioactivities in severe asthma. World Allergy Organ J 2016;9:21

4 Zeiger RS, Schatz M, Li Q, Chen W, Khatry DB, Gossage D, Tran TN: High blood eosinophil count is a risk factor for future asthma exacerbations in adult persistent asthma. J Allergy Clin Immunol Pract 2014;2:741-750.

5 Moore WC, Hastie AT, Li X, Li H, Busse WW, Jarjour NN, Wenzel SE, Peters SP, Meyers DA, Bleecker ER: Sputum neutrophil counts are associated with more severe asthma phenotypes using cluster analysis. J Allergy Clin Immunol 2014;133:1557-1563.e1555.

6 Rael EL, Lockey RF: Interleukin-13 signaling and its role in asthma. World Allergy Organ J 2011;4:54-64.

Tollip Inhibits Lung Eosinophilic Inflammation
7 Pope SM, Fulkerson PC, Blanchard C, Akei HS, Nikolaidis NM, Zimmermann N, Molkentin JD, Rothenberg ME: Identification of a cooperative mechanism involving interleukin-13 and eotaxin-2 in experimental allergic lung inflammation. J Biol Chem 2005; 280:13952-13961.

8 Pope SM, Brandt EB, Mishra A, Hogan SP, Zimmermann N, Matthaei KI, Foster PS, Rothenberg ME: IL-13 induces eosinophil recruitment into the lung by an IL-5- and eotaxin-dependent mechanism. J Allergy Clin Immunol 2001;108:594-601.

9 Jiang Z, Zhu L: Update on the role of alternatively activated macrophages in asthma. J Asthma Allergy 2016;9:101-107.

10 Siddiqui S, Secor ER Jr, Silbart LK: Bronchoalveolar macrophages express chemokines associated with leukocyte migration in a mouse model of asthma. Cell Immunol 2013;281: 159-169.

11 Balhara J, Gounni AS: The alveolar macrophages in asthma: a double-edged sword. $\mathrm{Mu}$ cosal Immunol 2012;5:605-609.
12 Capelluto DG: Tollip: a multitasking protein in innate immunity and protein trafficking. Microbes Infect 2012;14:140-147.

13 Shah JA, Vary JC, Chau TT, Bang ND, Yen NT, Farrar JJ, Dunstan SJ, Hawn TR: Human TOLLIP regulates TLR2 and TLR4 signaling and its polymorphisms are associated with susceptibility to tuberculosis. J Immunol 2012; 189:1737-1746.

14 Huang C, Jiang D, Francisco D, Berman R, Wu Q, Ledford JG, Moore CM, Ito Y, Stevenson C, Munson D, Li L, Kraft M, Chu HW: Tollip SNP rs5743899 modulates human airway epithelial responses to rhinovirus infection. Clin Exp Allergy 2016;46:1549-1563.

15 Moncayo-Nieto OL, Wilkinson TS, Brittan $\mathrm{M}$, McHugh BJ, Jones RO, Conway Morris A, Walker WS, Davidson DJ, Simpson AJ: Differential response to bacteria, and TOLLIP expression, in the human respiratory tract. BMJ Open Respir Res 2014;1:e000046. 
16 Song Z, Yin J, Yao C, Sun Z, Shao M, Zhang Y, Tao Z, Huang P, Tong C: Variants in the Toll-interacting protein gene are associated with susceptibility to sepsis in the Chinese Han population. Critical Care 2011;15:R12.

17 Noth I, Zhang Y, Ma SF, Flores C, Barber M, Huang Y, Broderick SM, Wade MS, Hysi P, Scuirba J, Richards TJ, Juan-Guardela BM, Vij R, Han MK, Martinez FJ, Kossen K, Seiwert SD, Christie JD, Nicolae D, Kaminski N, Garcia JG: Genetic variants associated with idiopathic pulmonary fibrosis susceptibility and mortality: a genome-wide association study. Lancet Respir Med 2013;1:309-317.

18 Schimming TT, Parwez Q, Petrasch-Parwez E, Nothnagel M, Epplen JT, Hoffjan S: Association of toll-interacting protein gene polymorphisms with atopic dermatitis. BMC Dermatol 2007;7:3.

19 Hollingsworth JW, Theriot BS, Li Z, Lawson BL, Sunday M, Schwartz DA, Walker JK: Both hematopoietic-derived and non-hematopoietic-derived $\beta$-arrestin- 2 regulates murine allergic airway disease. Am J Respir Cell Mol Biol 2010;43:269-275.

20 Kinyanjui MW, Shan J, Nakada EM, Qureshi ST, Fixman ED: Dose-dependent effects of IL-17 on IL-13-induced airway inflammatory responses and airway hyperresponsiveness. J Immunol 2013;190:3859-3868.

21 Chu HW, Honour JM, Rawlinson CA, Harbeck RJ, Martin RJ: Effects of respiratory $M y$ coplasma pneumoniae infection on allergeninduced bronchial hyperresponsiveness and lung inflammation in mice. Infect Immun 2003;71:1520-1526.

22 Kurowska-Stolarska M, Stolarski B, Kewin P, Murphy G, Corrigan CJ, Ying S, Pitman N, Mirchandani A, Rana B, van Rooijen N, Shepherd M, McSharry C, McInnes IB, Xu D, Liew FY: IL-33 amplifies the polarization of alternatively activated macrophages that contribute to airway inflammation. J Immunol 2009; 183:6469-6477.

23 Schmitz J, Owyang A, Oldham E, Song Y, Murphy E, McClanahan TK, Zurawski G, Moshrefi M, Qin J, Li X, Gorman DM, Bazan JF, Kastelein RA: IL-33, an interleukin-1-like cytokine that signals via the IL-1 receptor-related protein ST2 and induces T helper type 2-associated cytokines. Immunity 2005;23: 479-490.
24 Burns K, Clatworthy J, Martin L, Martinon F, Plumpton C, Maschera B, Lewis A, Ray K, Tschopp J, Volpe F: Tollip, a new component of the IL-1RI pathway, links IRAK to the IL-1 receptor. Nature Cell Biol 2000;2:346-351.

25 Yagami A, Orihara K, Morita H, Futamura K, Hashimoto N, Matsumoto K, Saito H, Matsuda A: IL-33 mediates inflammatory responses in human lung tissue cells. J Immunol 2010;185:5743-5750.

26 Nakae S, Morita H, Ohno T, Arae K, Matsumoto K, Saito H: Role of interleukin-33 in innate-type immune cells in allergy. Allergol Int 2013;62:13-20.

27 Ohno T, Oboki K, Kajiwara N, Morii E, Aozasa K, Flavell RA, Okumura K, Saito H, Nakae S: Caspase-1, caspase-8, and calpain are dispensable for IL-33 release by macrophages. J Immunol 2009;183:7890-7897.

28 Nabe T, Wakamori H, Yano C, Nishiguchi A, Yuasa R, Kido H, Tomiyama Y, Tomoda A, Kida H, Takiguchi A, Matsuda M, Ishihara K, Akiba S, Ohya S, Fukui H, Mizutani N, Yoshino S: Production of interleukin (IL)-33 in the lungs during multiple antigen challengeinduced airway inflammation in mice, and its modulation by a glucocorticoid. Eur J Pharmacol 2015;757:34-41.

29 Ohno T, Oboki K, Morita H, Kajiwara N, Arae $\mathrm{K}$, Tanaka S, Ikeda M, Iikura M, Akiyama T, Inoue J, Matsumoto K, Sudo K, Azuma M, Okumura K, Kamradt T, Saito H, Nakae S: Paracrine IL-33 stimulation enhances lipopolysaccharide-mediated macrophage activation. PLoS One 2011;6:e18404

30 Joshi AD, Oak SR, Hartigan AJ, Finn WG, Kunkel SL, Duffy KE, Das A, Hogaboam CM: Interleukin-33 contributes to both $\mathrm{M} 1$ and M2 chemokine marker expression in human macrophages. BMC Immunol 2010;11:52.

31 Saha SS, Singh D, Raymond EL, Ganesan R, Caviness G, Grimaldi C, Woska JR Jr, Mennerich D, Brown SE, Mbow ML, Kao CC: Signal transduction and intracellular trafficking by the interleukin 36 receptor. J Biol Chem 2015;290:23997-24006.
32 Brissoni B, Agostini L, Kropf M, Martinon F, Swoboda V, Lippens S, Everett H, Aebi N, Janssens S, Meylan E, Felberbaum-Corti M, Hirling H, Gruenberg J, Tschopp J, Burns K: Intracellular trafficking of interleukin-1 receptor I requires Tollip. Curr Biol 2006;16: 2265-2270.

33 Zhu L, Wang L, Luo X, Zhang Y, Ding Q, Jiang X, Wang X, Pan Y, Chen Y: Tollip, an intracellular trafficking protein, is a novel modulator of the transforming growth factor- $\beta$ signaling pathway. J Biol Chem 2012; 287:39653-39663.

34 Cayrol C, Girard JP: IL-33: an alarmin cytokine with crucial roles in innate immunity, inflammation and allergy. Curr Opin Immunol 2014;31:31-37.

35 Liew FY, Girard JP, Turnquist HR: Interleukin-33 in health and disease. Nat Rev Immunol 2016;16:676-689.

36 Traister RS, Uvalle CE, Hawkins GA, Meyers DA, Bleecker ER, Wenzel SE: Phenotypic and genotypic association of epithelial IL1RL1 to human TH2-like asthma. J Allergy Clin Immunol 2015;135:92-99.

37 Christianson CA, Goplen NP, Zafar I, Irvin C, Good JT Jr, Rollins DR, Gorentla B, Liu W, Gorska MM, Chu H, Martin RJ, Alam R: Persistence of asthma requires multiple feedback circuits involving type 2 innate lymphoid cells and IL-33. J Allergy Clin Immunol 2015;136: 59-68.e14.

38 Kurowska-Stolarska M, Kewin P, Murphy G, Russo RC, Stolarski B, Garcia CC, Komai-Koma M, Pitman N, Li Y, Niedbala W, McKenzie AN, Teixeira MM, Liew FY, Xu D: IL-33 induces antigen-specific IL-5+ T cells and promotes allergic-induced airway inflammation independent of IL-4. J Immunol 2008; 181 : 4780-4790.

39 Smith SG, Chen R, Kjarsgaard M, Huang C, Oliveria JP, O’Byrne PM, Gauvreau GM, Boulet LP, Lemiere C, Martin J, Nair P, Sehmi R: Increased numbers of activated group 2 innate lymphoid cells in the airways of patients with severe asthma and persistent airway eosinophilia. J Allergy Clin Immunol 2016;137: 75-86.e78. 